\title{
Cerebral mechanisms of language: magnetoencephalographic studies Andrew Papanicolaou*
}

Address: Division of Clinical Neurosciences, Department of Neurosurgery, University of Texas-Houston Medical School, USA

* Corresponding author

from International Society on Brain and Behaviour: 2nd International Congress on Brain and Behaviour Thessaloniki, Greece. 17-20 November 2005

Published: 28 February 2006

Annals of General Psychiatry 2006, 5(Suppl I):S32 doi:I0.I I86/I744-859X-5-SI-S32

\begin{abstract}
A series of studies involving the use of Magnetoencephalography and aiming to specify the cerebral mechanisms of language will be reviewed. The series consists, first, of studies establishing the reliably, validity, and the degree of spatiotemporal detail achievable through MEG-mapping; second of studies exploring the brain mechanisms of oral language comprehension, specifically the mechanisms mediating phonological and semantic processing of speech; third, those exploring the mechanisms mediating verbal and non-verbal memory and reading in normal children and adults. Finally, a fourth set of studies will be reviewed focusing on the gradual changes of these language-specific mechanisms during child development, on the discovery of aberrant spatiotemporal brain activation profiles associated with dyslexia, and on the restitution of normal brain activity patterns following successful remedial training in reading testifying to the enormous capacity of the brain for behaviorally-induced functional reorganization.
\end{abstract}

